Mathematical Problems of Computer Science 49, 35-40, 2018.

\title{
Degree Sequences and Dominating Cycles in 2-Connected Graphs
}

\author{
Mossine S. Koulakzyan \\ Institute for Informatics and Automation Problems of NAS RA \\ e-mail: zhora@ipia.sci.am
}

\begin{abstract}
Let $G$ be a graph on $n$ vertices and minimum degree $\delta$ with degree sequence $\delta=$ $d_{1} \leq d_{2} \leq \ldots \leq d_{n}$. The minimum degree sum of two nonadjacent vertices in $G$ is denoted by $\sigma_{2}$. Let $c$ be the circumference - the order (the number of vertices) of a longest cycle, and $p$ be the order of a longest path in $G$. In 1952, Dirac proved: (1) if $G$ is a 2-connected graph, then $c \geq \min \left\{n, 2 d_{1}\right\}$; (2) every graph with $d_{1} \geq \frac{n}{2}$ is hamiltonian. Recently, these results were improved by Nikoghosyan in terms of degree sequences: (3) if $G$ is a 2-connected graph, then $c \geq \min \left\{n, d_{\delta}+d_{\delta+1}\right\}$; (4) every graph with $d_{\delta}+d_{\delta+1} \geq n$ is hamiltonian. In this paper we present the dominating cycle versions of these theorems: (i) if $G$ is a 2-connected graph, then either $c \geq d_{\delta}+d_{\sigma_{2}}$ or $c \geq p-1$ (that is $G$ has a dominating cycle); (ii) every 2-connected graph with $d_{\delta}+d_{\delta+1} \geq p-1$ has a dominating cycle. The results are sharp.
\end{abstract}

Keywords: Hamilton cycle, dominating cycle, circumference, minimum degree, degree sums, degree sequence.

\section{Introduction}

We consider only finite undirected graphs with neither loops nor multiple edges. A good reference for any undefined terms is [1].

The set of vertices of a graph $G$ is denoted by $V(G)$, and the set of edges by $E(G)$. Let $n$ be the order (the number of vertices) of $G, c$ the order of a longest cycle (called circumference) in $G$ and $p$ the order of a longest path. The minimum degree sum of two nonadjacent vertices in $G$ is denoted by $\sigma_{2}$. In particular, the minimum degree in $G$ is denoted by $\delta$. Let $d_{1}, d_{2}, \ldots, d_{n}$ be the degree sequence in $G$ with $\delta=d_{1} \leq d_{2} \leq \ldots \leq d_{n}$. We use $N(v)$ to denote the set of all neighbors of a vertex $v$ and $d(v)=|N(v)|$ to denote the degree of vertex $v$. A graph $G$ is hamiltonian if $G$ contains a Hamilton cycle, that is a simple spanning cycle. A cycle $C$ of $G$ is called a dominating cycle if every edge of $G$ has at least one of its end vertices on $C$, or, equivalently, if $G-V(C)$ contains no edges.

We write a cycle $Q$ with a given orientation by $\vec{Q}$. For $x, y \in V(Q)$, we denote by $x \vec{Q} y$ the subpath of $Q$ in the chosen direction from $x$ to $y$. For $x \in V(Q)$, we denote the successor and the predecessor of $x$ on $\vec{Q}$ (if such vertices exist) by $x^{+}$and $x^{-}$, respectively. For $U \subseteq V(Q)$, we denote $U^{+}=\left\{u^{+} \mid u \in U\right\}$ and $U^{-}=\left\{u^{-} \mid u \in U\right\}$. We say that the vertex 
$z_{1}$ precedes the vertex $z_{2}$ on a path $\vec{Q}$ if $z_{1}, z_{2}$ occur on $\vec{Q}$ in this order and indicate this relationship by $z_{1} \prec z_{2}$. We will write $z_{1} \preceq z_{2}$ when either $z_{1}=z_{2}$ or $z_{1} \prec z_{2}$.

Let $\vec{P}=v_{1} v_{2} \ldots v_{p}$ be a longest path in $G$. Clearly, $N\left(v_{1}\right) \cup N\left(v_{p}\right) \subseteq V(P)$. A vine of length $m$ on $P$ is a set

$$
\left\{L_{i}=w_{i} \vec{L}_{i} z_{i}: 1 \leq i \leq m\right\}
$$

of internally-disjoint paths such that

(a) $V\left(L_{i}\right) \cap V(P)=\left\{w_{i}, z_{i}\right\} \quad(i=1, \ldots, m)$,

(b) $v_{1}=w_{1} \prec w_{2} \prec z_{1} \preceq w_{3} \prec z_{2} \preceq w_{4} \prec \ldots \preceq w_{m} \prec z_{m-1} \prec z_{m}=v_{p}$ on $P$.

The following result guarantees the existence of at least one vine on $\vec{P}$ in a 2-connected graph.

The Vine Lemma [2]. If $G$ is a 2-connected graph and $P$ a path in $G$, then there is at least one vine on $P$.

In 1952, Dirac [2] obtained the first lower bound for the circumference for 2-connected graphs and the first sufficient condition for Hamilton cycles in terms of minimum degree $\delta$.

Theorem A [2]. If $G$ is a 2-connected graph, then $c \geq \min \{n, 2 \delta\}=\min \left\{n, 2 d_{1}\right\}$.

Theorem B [2]. Every graph with $\delta=d_{1} \geq \frac{n}{2}$ is hamiltonian.

Theorems A and B were improved in [3] in terms of degree sequences.

Theorem $\mathbf{C}[3]$. If $G$ is a 2-connected graph, then $c \geq \min \left\{n, d_{\delta}+d_{\delta+1}\right\}$.

Theorem D [3]. Every graph with $d_{\delta}+d_{\delta+1} \geq n$ is hamiltonian.

In this paper we present the dominating versions of Theorems $\mathrm{C}$ and D.

Proposition 1 [4]. Let $G$ be a connected graph with $c \geq p-1$. Then every longest cycle in $G$ is a dominating cycle.

Theorem 1. If $G$ is a 2-connected graph, then $c \geq \min \left\{p-1, d_{\delta}+d_{\sigma_{2}}\right\}$.

The next result follows from Theorem 1 immediately as a sufficient condition for the existence of a dominating cycle.

Theorem 2. If $G$ is a 2-connected graph with $d_{\delta}+d_{\sigma_{2}} \geq p-1$, then $c \geq p-1$.

If $G=\bar{K}_{\delta+1}+K_{\delta}$, then $d_{\delta}=\delta, d_{2 \delta}=d_{\sigma_{2}}=2 \delta=\sigma_{2}$ and $c=2 \delta=\sigma_{2}=p-1$. This graph example shows that the conclusion "either $c \geq d_{\delta}+d_{\sigma_{2}}$ or $c \geq p-1$ " in Theorem 3 cannot be replaced by "either $c \geq d_{\delta}+d_{\sigma_{2}}$ or $c \geq p$ ". Next, let $G=\delta K_{2}+K_{\delta-1}$. Then $d_{\delta}=d_{2 \delta}=d_{\sigma_{2}}=\delta, d_{2 \delta+1}=d_{\sigma_{2}+1}=3 \delta-2$ and $c=3 \delta-3=p-2$. This graph example shows that the conclusion " either $c \geq d_{\delta}+d_{\sigma_{2}}$ or $c \geq p-1$ " in Theorem 1 cannot be replaced 
by "either $c \geq d_{\delta}+d_{\sigma_{2}+1}$ or $c \geq p-1$ ". Thus, Theorem 1 is best possible.

\section{Proofs}

Proof of Theorem 1. Let $\vec{P}=v_{1} v_{2} \ldots v_{p}$ be a longest path in $G$. Clearly,

$$
N\left(v_{1}\right) \cup N\left(v_{p}\right) \subseteq V(P) .
$$

Assume that

(a1) $P$ is chosen so that $d\left(v_{1}\right)$ is maximum.

(a2) $P$ is chosen so that $d\left(v_{p}\right)$ is maximum subject to $(a 1)$.

Let $x_{1}, x_{2}, \ldots, x_{t}$ be the elements of $N\left(v_{1}\right)$ occurring on $\vec{P}$ in a consecutive order, where $t=d\left(v_{1}\right) \geq \delta$. Next, let $y_{1}, y_{2}, \ldots, y_{f}$ be the elements of $N\left(v_{p}\right)$ occurring on $\overleftarrow{P}$ in a consecutive order. If either $x_{t}=v_{p}$ or $y_{f}=v_{1}$, then we can form a path longer than $P$, a contradiction. Hence, $x_{t} \neq v_{p}$ and $y_{f} \neq v_{1}$. Observe that for each $i \in\{1,2, \ldots, t\}$,

$$
x_{i}^{-} \overleftarrow{P} v_{1} x_{i} \vec{P} v_{p}
$$

is a longest path in $G$, implying that

$$
N\left(x_{i}^{-}\right) \subseteq V(P) \quad(i=1,2, \ldots, t) .
$$

By a symmetric argument,

$$
N\left(y_{i}^{+}\right) \subseteq V(P) \quad(i=1,2, \ldots, f) .
$$

Case 1. $x_{t} \preceq y_{f}$.

Let

$$
\left\{L_{i}=w_{i} \vec{L}_{i} z_{i}: 1 \leq i \leq m\right\}
$$

be a vine of minimal length $m$ on $\vec{P}$. Since $P$ is a longest path in $G$, we have $L_{1}, L_{M} \in E(G)$. Next, since $m$ is minimal, we have $x_{t} \prec z_{2}, x_{t} \prec w_{3}$ and $w_{m-1} \prec y_{f}, z_{m-2} \prec y_{f}$. Choose $z_{1}^{*} \in V(P)$ such that $w_{2} \prec z_{1}^{*}$ and $\left|V\left(w_{2} \vec{P} z_{1}^{*}\right)\right|$ is minimal. Analogously, choose $w_{m}^{*} \in V(P)$ such that $w_{m}^{*} \prec z_{m-1}$ and $\left|V\left(w_{m}^{*} \vec{P} z_{m-1}\right)\right|$ is minimal. Put

$$
H=P \cup \bigcup_{i=2}^{m-1} L_{i} \cup\left\{v_{1} z_{1}^{*}, v_{p} w_{m}^{*}\right\} .
$$

By deleting the following paths

$$
w_{i} \vec{P} z_{i-1} \quad(i=3,4, \ldots, m-1), \quad w_{2} \vec{P} z_{1}^{*}, \quad w_{m}^{*} \vec{P} z_{m-1}
$$

from $H$ (except for their endvertices), we obtain a cycle $C$ with at least $d\left(v_{1}\right)+d\left(v_{p}\right)+1$ vertices. Since the vertices $x_{1}^{-}, x_{2}^{-}, \ldots, x_{t}^{-}, y_{1}^{+}, y_{2}^{+}, \ldots, y_{f}^{+}$are pairwise distinct, we have

$$
d\left(v_{1}\right)=\max \left\{d\left(x_{1}^{-}\right), d\left(x_{2}^{-}\right), \ldots, d\left(x_{t}^{-}\right), d\left(y_{1}^{+}\right), d\left(y_{2}^{+}\right), \ldots, d\left(y_{f}^{+}\right)\right\}
$$




$$
\begin{gathered}
\geq \max \left\{d_{1}, d_{2}, \ldots, d_{t+f}\right\}=d_{t+f}=d_{d\left(v_{1}\right)+d\left(v_{p}\right)} \geq d_{\sigma_{2}}, \\
d\left(v_{p}\right)=\max \left\{d\left(y_{1}^{+}\right), d\left(y_{2}^{+}\right), \ldots, d\left(y_{f}^{+}\right)\right\} \geq \max \left\{d_{1}, d_{2}, \ldots, d_{f}\right\}=d_{f}=d_{d\left(v_{p}\right)} \geq d_{\delta},
\end{gathered}
$$

implying that

$$
c \geq d\left(v_{1}\right)+d\left(v_{p}\right)+1>d_{\delta}+d_{\sigma_{2}} .
$$

Case 2. $y_{f} \prec x_{t}$.

Case 2.1. $N\left(v_{1}\right) \cap N^{+}\left(v_{p}\right) \neq \emptyset$.

Let $v \in N\left(v_{1}\right) \cap N^{+}\left(v_{p}\right)$, that is $v_{1} v, v_{p} v^{-} \in E(G)$. Since

$$
v_{1} v \vec{P} v_{p} v^{-\overleftarrow{P}} v_{1}
$$

is a cycle of order $p$, and $G$ is connected, either $p<|V(G)|$, and we can form a path longer than $P$ (a contradiction) or $p=|V(G)|$, implying that $c=p$.

Case 2.2. $N\left(v_{1}\right) \cap N^{+}\left(v_{p}\right)=\emptyset$.

Case 2.2.1. $N^{-}\left(v_{1}\right) \cap N^{+}\left(v_{p}\right) \neq \emptyset$.

Let $v \in N^{-}\left(v_{1}\right) \cap N^{+}\left(v_{p}\right)$, that is $z=x_{i}^{-}=y_{j}^{+}$for some $i \in\{1, \ldots, t\}$ and $j \in\{1, \ldots, f\}$. Clearly,

$$
v_{1} z^{+} \vec{P} v_{p} z^{-\overleftarrow{P}} v_{1}
$$

is a cycle of order $p-1$, that is $c \geq p-1$.

Case 2.2.2. $N^{-}\left(v_{1}\right) \cap N^{+}\left(v_{p}\right)=\emptyset$.

Since $y_{f} \prec x_{t}$, we can choose two integers $1 \leq a \leq t$ and $1 \leq b \leq f$ such that $y_{b} \prec x_{a}$, and $\left|V\left(y_{b} \vec{P} x_{p}\right)\right|$ is minimum. Put

$$
C=v_{1} x_{a} \vec{P} v_{p} y_{b} \overleftarrow{P} v_{1}
$$

Clearly,

$$
\left(N\left(v_{1}\right) \cup N^{+}\left(v_{p}\right)\right)-y_{b}^{+} \subseteq V(C) .
$$

Hence,

$$
\begin{gathered}
c \geq|V(C)| \geq\left|\left(N\left(v_{1}\right) \cup N^{+}\left(v_{p}\right)\right)-y_{b}^{+}\right|+\left|\left\{v_{1}\right\}\right| \\
=\left|N\left(v_{1}\right)\right|+\left|N^{+}\left(v_{p}\right)\right|=\left|N\left(v_{1}\right)\right|+\left|N\left(v_{p}\right)\right|=d\left(v_{1}\right)+d\left(v_{p}\right) .
\end{gathered}
$$

By the hypothesis, the following vertices

$$
x_{1}^{-}, x_{2}^{-}, \ldots, x_{t}^{-}, y_{1}^{+}, y_{2}^{+}, \ldots, y_{f}^{+}
$$

are pairwise distinct. By (a1) and (a2),

$$
\begin{gathered}
d\left(v_{1}\right)=\max \left\{d\left(x_{1}^{-}\right), d\left(x_{2}^{-}\right), \ldots, d\left(x_{t}^{-}\right), d\left(y_{1}^{+}\right), d\left(y_{2}^{+}\right), \ldots, d\left(y_{f}^{+}\right)\right\} \\
\geq \max \left\{d_{1}, d_{2}, \ldots, d_{t+f}\right\}=d_{t+f}=d_{d\left(v_{1}\right)+d\left(v_{p}\right)} \geq d_{\sigma_{2}}, \\
d\left(v_{p}\right)=\max \left\{d\left(y_{1}^{+}\right), d\left(y_{2}^{+}\right), \ldots, d\left(y_{f}^{+}\right)\right\} \\
\geq \max \left\{d_{1}, d_{2}, \ldots, d_{f}\right\}=d_{f}=d_{d\left(v_{p}\right)} \geq d_{\delta},
\end{gathered}
$$

implying that

$$
c \geq d\left(v_{1}\right)+d\left(v_{p}\right) \geq d_{\delta}+d_{\sigma_{2}} .
$$




\section{References}

[1] J. A. Bondy and U. S. R. Murty, Graph Theory with Applications, Macmillan, London and Elsevier, New York 1976.

[2] G. A. Dirac, "Some theorems on abstract graphs", Proc. London, Math. Soc., vol. 2, pp. 69-81, 1952.

[3] Zh. G. Nikoghosyan, "Degree sequences and long cycles in Graphs", ArXiv:1711.04134 (2017) 9 pages.

[4] K. Ozeki and T. Yamashita, "Length of longest cycles in a graph whose relative length is at least two", Graphs and Combin., vol. 28, pp. 859-868, 2012.

Submitted 22.08.2017, accepted 14.12.2017.

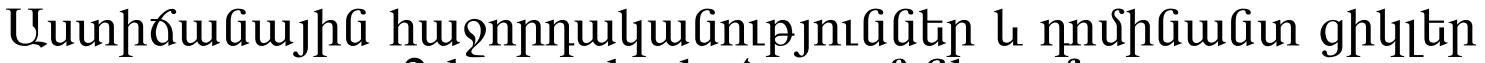 2-luuuulgumo qnuфGitipnı}

U. คnıцuрquшi

\section{Ưưnนhnน์}

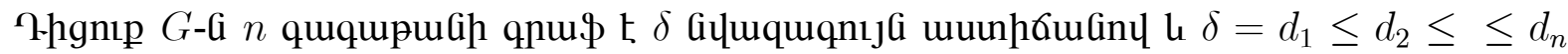

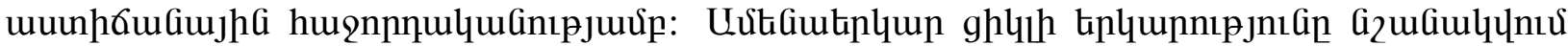

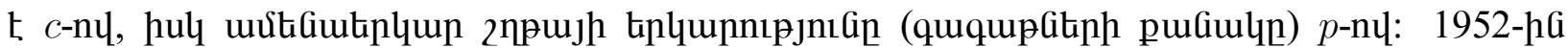

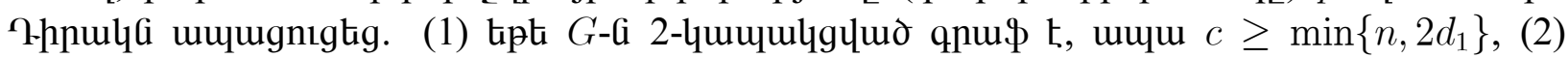

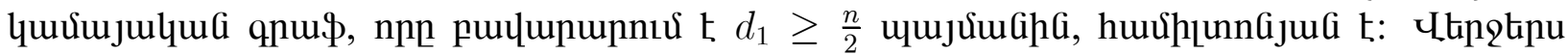

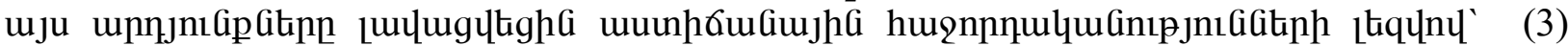

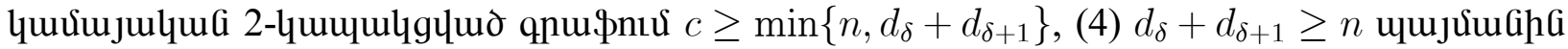

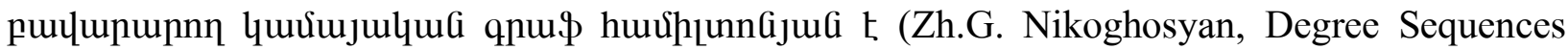
and Long Cycles in Graphs, ArXiv:1711.04134): Ghplu uzhumunuGpnıu phplntu tia (3) l

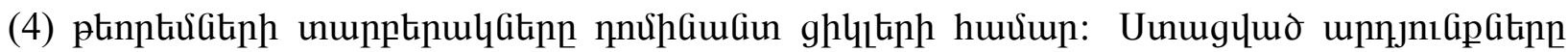
julumgGith stig: 


\section{Степенные последовательности и доминантные циклы в 2-связных графах}

М. Кулакзян

\section{Аннотация}

Пусть $G$ является $n$ вершинным графом с минимальной степенью $\delta$ и степенной последовательностью $\delta=d_{1} \leq d_{2} \leq \leq d_{n}$. Алина Алиннейшего цикла обозначается через $c$, а Алина длиннейшей цепи (число её вершин) через $p$. В 1952 году Аирак доказал: (1) если $G$ является 2-связным графом, то $c \geq \min \left\{n, 2 d_{1}\right\}$; (2) если граф удовлетворяет условию $d_{1} \geq \frac{n}{2}$, то он является гамильтоновым. Недавно эти результаты были улучшены в терминах степенных последовательностей: (3) если $G$ является 2-связным графом, то $c \geq \min \left\{n, d_{\delta}+\right.$ $\left.d_{\delta+1}\right\} ;$ (4) если граф удовлетворяет условию $d_{\delta}+d_{\delta+1} \geq n$, то он является гамильтоновым (Zh.G. Nikoghosyan, Degree Sequences and Long Cycles in Graphs, ArXiv:1711.04134). В настоящей работе представляются версии теорем (3) и (4) для доминантных циклов. Полученные результаты неулучшаемы. 